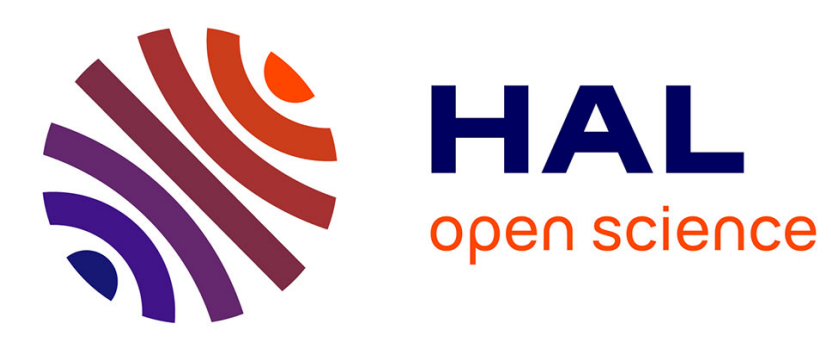

\title{
Effects of slip on steady Bödewadt flow of a non-Newtonian fluid
}

Bikash Sahoo, Sébastien Poncet

\section{To cite this version:}

Bikash Sahoo, Sébastien Poncet. Effects of slip on steady Bödewadt flow of a nonNewtonian fluid. Communication Nonlinear Science Numerical Simulation, 2012, 17, pp.4181-4191. 10.1016/j.cnsns.2012.02.031 . hal-00822028

\section{HAL Id: hal-00822028 \\ https://hal.science/hal-00822028}

Submitted on 13 May 2013

HAL is a multi-disciplinary open access archive for the deposit and dissemination of scientific research documents, whether they are published or not. The documents may come from teaching and research institutions in France or abroad, or from public or private research centers.
L'archive ouverte pluridisciplinaire HAL, est destinée au dépôt et à la diffusion de documents scientifiques de niveau recherche, publiés ou non, émanant des établissements d'enseignement et de recherche français ou étrangers, des laboratoires publics ou privés. 


\title{
Effects of slip on steady Bödewadt flow of a non-Newtonian fluid
}

\author{
Bikash Sahoo \\ Department of Mathematics, National Institute of Technology, Rourkela, Orissa, \\ INDIA - Tel. +916612462706 \\ Sébastien Poncet \\ Laboratoire M2P2 UMR 7340 CNRS - Aix-Marseille Université, Marseille, \\ FRANCE - Tel. +33 491118555
}

\begin{abstract}
The steady flow arising due to the rotation of a non-Newtonian fluid at a larger distance from a stationary disk is extended to the case where the disk surface admits partial slip. The constitutive equation of the non-Newtonian fluid is modeled by that for a Reiner-Rivlin fluid. The momentum equation gives rise to a highly nonlinear boundary value problem. Numerical solution of the governing nonlinear equations are obtained over the entire range of the physical parameters. The effects of slip and non-Newtonian fluid characteristics on the momentum boundary layer are discussed in details. It is observed that slip has prominent effect on the velocity field, whereas a predominant influence of the non-Newtonian parameter is observed on the moment coefficient.
\end{abstract}

Key words: Reiner-Rivlin fluid, Bödewadt flow, Partial slip, Finite difference method.

PACS: 47.15, 47.50, 47.65

\section{Introduction}

The flow of non-Newtonian fluid due to a rotating disk has received much attention during the last decades [1-3] due to its immense industrial applications

Email addresses: bikashsahoo@nitrkl.ac.in (Bikash Sahoo), poncet@13m.univ-mrs.fr (Sébastien Poncet).

Preprint submitted to Applied Mathematical Modelling 
such as for designing gaseous core nuclear reactor or power generators, increasing storage density in hard-disk drives and some applications in rheology of course. In previous works [4,5], we have discussed the steady Von Kármán flow and heat transfer of an electrically conducting Reiner-Rivlin fluid with partial slip boundary conditions. The twin problem arising when the fluid rotates with an uniform angular velocity at a larger distance from a stationary disk is one of the classical problems of fluid mechanics which has both theoretical and practical value. In this case, the particles which rotate at a large distance from the wall are in equilibrium under the influence of the centrifugal force which is balanced by the radial pressure gradient. Those particles close to the disk whose circumferential velocity is retarded under the same pressure gradient directed inwards. However, the centrifugal force they are subjected to is greatly decreased. This set of circumstances causes the particle near the disk to flow radially inwards, and for reasons of continuity that motion must be compensated by an axial flow upwards, as shown in Figure 1. A flow which arises in the boundary layer in this manner such that its direction deviates from that of the outer flow is generally called a secondary flow. Such type of flow can be clearly observed in a teacup: after the rotation has been generated by vigorous stirring and again after the flow has been left to itself for a short while. The radial inward flow field near the bottom will be formed. Its existence can be inferred from the fact that tea leaves settle in a little heap near the center at the bottom.

This problem was studied by Bödewadt [6] by making boundary layer approximations. That is why the flow problem is widely known as the Bödewadt flow. For this problem Ackroyed's method [7] of expansion is not so suitable. Bödewadt approached the solution through a very laborious method similar to that used by Cochran [8] for the Von Kármán equations. The method consists of a power series expansion at $z=0$ and an asymptotic expansion for $z \rightarrow \infty$. Bödewadt's solution shows that the boundary layer effects extend out to about $\zeta=8.0$, where $\zeta$ is the non-dimensional distance measured along the rotation axis. A correction to this problem is worked out by Browning (unpublished, see in [9]). He noticed a much thicker boundary layer than in the case of a disk rotating in a fluid at rest. Batchelor [10] generalized theses analysis to include one parameter families of solutions having a mathematical structure very similar to that of Von Kármán's. It corresponds to the flow above an infinite disk rotating with certain angular velocity, with the fluid in the far off region in solid body rotation. The general problem of an infinite rotating fluid of which the above two problems are particular cases has been later investigated by Hannah [11]. Subsequently, this case has been treated by Stewartson [12]. Fettis [13] had been concerned largely with the problem of Bödewadt. Rogers and Lance [14] studied numerically a similar problem when the disk rotates with an angular velocity $\omega$ in a fluid rotating with a different speed $\Omega$. When $\Omega=0$, the system reduces to the free-disk problem of Von Kármán; when $\omega=\Omega$, there is a solid-body rotation; and 
when $\omega=0$, the problem is the same as that discussed by Bödewadt. It was pointed out by Schwiderski and Lugt [15] that the non-existence of a proper solution to the boundary value problems for rotating flows of Von Kármán and Bödewadt is an indication that in reality the flow is separated from the surface of the disk. The simple 'Tea cup experiment' described above, displays very clearly a separation of the fluid from the bottom of the cup. Application of the suction is an effective device to reduce the chances of separation. Later, the local boundary layer approximations of first order derived in [15] have been generalized by Schwiderski and Lugt [16] to axisymmetric motions which rotates over a rotating disk of infinite dimensions. Numerical results are computed and discussed for a variety of Reynolds numbers and for cases for which the disk is rotating in the same sense and in the opposite sense as the fluid far away from the disk. The critical Reynolds numbers for steady laminar motions which are attached to the surface of the disk are computed and displayed. Nanda [17] studied the effects of uniform suction on the revolving flow of a viscous liquid over a stationary disk. It was found that the presence of suction introduces an axial inflow at infinity and the same increases with an increase in suction. Nydahl [18] in his doctoral thesis has extended the Bödewadt flow problem by incorporating the heat transfer phenomena. The results obtained by Nydahl effectively confirm those of Bödewadt; those of Rogers and Lance [19] give a significantly larger value of $H_{\infty}$. The momentum and the displacement thickness decrease as the suction velocity increases. The spin-up process in the Bödewadt flow of a viscous fluid has been studied by Chawla and Purushothaman [20]. A comprehensive review of earlier works on flow and heat transfer due to a single and two parallel rotating disks up to 1989 has been included in a monograph by Owen and Rogers [21]. Recently, Chawla and Srivastava [22] have considered the physical situation in which the disk is performing torsional oscillations in contact with a fluid in a state of rigid-body rotation in the far field. Sharon and MacKerrel [23] have studied the stability of Bödewadt flow. Kitchens and Chang [24] have considered the Bödewadt flow for a non-Newtonian second-order fluid.

A literature survey indicates that there has been an extensive literature available regarding the boundary layer flow over a rotating disk (Kármán flow) in various situations. Such studies include different fluid models, magnetohydrodynamic and hydrodynamic cases, with and without heat transfer analysis. As can be seen from the literature, there has been relatively little information regarding the Bödewadt type flow. The present study is an endeavor to fill this gap. An important application of such flow arises from modelling atmospheric flows. Besides the above reason, few other curious findings which motivated for the present investigation are as follows.

First of all the steady Bödewadt flow of a viscous, incompressible fluid is one of the few problems in fluid dynamics for which the Navier-Stokes equations admit an exact solution. Again as reported by Owen and Rogers [21], there is 
a discrepancy in the numerical value of $H_{\infty}$ (axial flow velocity at sufficiently large distance from the surface of the disk), which was found to be 1.3494 using the method of Rogers and Lance [14] on a VAX 8530 computer with a typical precision of 16 significant figures. This depicts that the accuracy achieved by Rogers and Lance [14] was not sufficient to give an accurate value of $H_{\infty}$. Moreover, it has been pointed out by many authors that Bödewadt's solution implies that there is a flow out of the boundary layer everywhere and no mechanism for supplying fluid to it! For an infinite disk, the problem may be overcome by assuming an infinite reservoir of fluid from which the boundary layer can draw in an unspecified way. For the more practical case of a finite disk, it must be supposed that a similarity solution does not hold near the edge of the disk. However, it is consistent with experience in other fields that a similarity solution becomes valid as the boundary layer flow develops. It may then be assumed that fluid enters the boundary layer near the edge of the disk and that this fluid is available for continuity in the similarity solution.

This paper is divided as follows: in Section 2, the problem is mathematically formulated to get a system of ordinary differential equations with appropriate boundary conditions and is solved using an effective second order numerical scheme in Section 3. The effects of the partial slip and the non-Newtonian cross-viscous parameters on the momentum boundary layer are discussed in details in Section 4 before some concluding remarks in Section 5.

\section{Flow analysis}

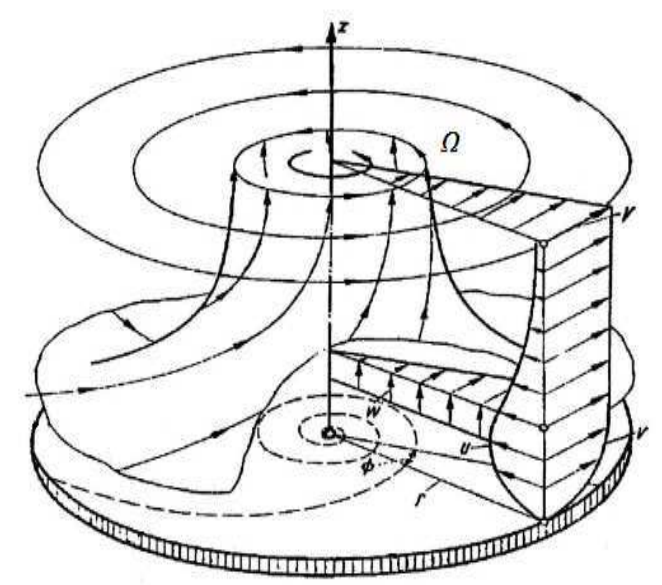

Fig. 1. Schematic representation of the Bödewadt flow after Schlichting [9].

In this case, we consider the non-Newtonian Reiner-Rivlin fluid where the stress tensor $\tau_{i j}$ is related to the rate of strain tensor $e_{i j}[3]$ as: 


$$
\begin{array}{r}
\tau_{i j}=-p \delta_{i j}+2 \mu e_{i j}+2 \mu_{c} e_{i k} e_{k j} \\
e_{j j}=0
\end{array}
$$

where $p$ is denoting the pressure, $\mu$ is the coefficient of viscosity and $\mu_{c}$ is the coefficient of cross-viscosity.

The fluid occupies the space $z>0$ over an infinite stationary disk (Fig. 1), which coincides with $z=0$. The motion is due to the rotation of the fluid like a rigid body with constant angular velocity $\Omega$ at large distance from the disk.

The flow is described in the cylindrical polar coordinates $(r, \phi, z)$. In view of the rotational symmetry $\frac{\partial}{\partial \phi} \equiv 0$. The no-slip boundary conditions for the velocity field are given as

$$
\begin{aligned}
& z=0, \quad u=0, \quad v=0, \quad w=0 \\
& z \rightarrow \infty, \quad u \rightarrow 0, \quad v \rightarrow r \Omega, \quad p \rightarrow p_{\infty} .
\end{aligned}
$$

The Von Kármán transformations [25]

$u=r \Omega F(\zeta), \quad v=r \Omega G(\zeta), \quad w=\sqrt{\Omega \nu} H(\zeta), \quad z=\sqrt{\frac{\nu}{\Omega}} \zeta, \quad p-p_{\infty}=-\rho \nu \Omega P$

reduce the Navier-Stokes equations for a Newtonian fluid to a set of ordinary differential equations. The same is also true for a non-Newtonian Reiner-Rivlin fluid. We define the non-Newtonian cross-viscous parameter $L=\frac{\mu_{c} \Omega}{\mu}$. With these definitions and by considering the usual boundary layer approximations, the equations of continuity and motion take the form,

$$
\begin{aligned}
& \frac{d H}{d \zeta}+2 F=0 \\
& \frac{d^{2} F}{d \zeta^{2}}-H \frac{d F}{d \zeta}-F^{2}+G^{2}-F-\frac{1}{2} L\left[\left(\frac{d F}{d \zeta}\right)^{2}-3\left(\frac{d G}{d \zeta}\right)^{2}-2 F \frac{d^{2} F}{d \zeta^{2}}\right]=1, \\
& \frac{d^{2} G}{d \zeta^{2}}-H \frac{d G}{d \zeta}-2 F G-G+L\left(\frac{d F}{d \zeta} \frac{d G}{d \zeta}+F \frac{d^{2} G}{d \zeta^{2}}\right)=0 \\
& \frac{d^{2} H}{d \zeta^{2}}-H \frac{d H}{d \zeta}-\frac{7}{2} L \frac{d H}{d \zeta} \frac{d^{2} H}{d \zeta^{2}}+\frac{d P}{d \zeta}=0
\end{aligned}
$$

and the boundary conditions (3) become,

$$
\begin{aligned}
& \zeta=0: \quad F=0, \quad G=0, \quad H=0 \\
& \zeta \rightarrow \infty: \quad F \rightarrow 0, \quad G \rightarrow 1 .
\end{aligned}
$$

The fluid adheres to the surface partially and the motion of the fluid exhibits partial slip conditions. A generalization of Navier's partial slip condition gives, in the radial direction,

$$
\left.u\right|_{z=0}=\left.\lambda_{1} T_{r z}\right|_{z=0},
$$


and in the azimuthal direction

$$
\left.v\right|_{z=0}=\left.\lambda_{2} T_{\phi z}\right|_{z=0}
$$

where $\lambda_{1}, \lambda_{2}$ are respectively the slip coefficients, and $T_{r z}, T_{\phi z}$ are the physical components of the stress tensor. Let

$$
\lambda=\lambda_{1} \sqrt{\frac{\Omega}{\nu}} \mu, \quad \eta=\lambda_{2} \sqrt{\frac{\Omega}{\nu}} \mu .
$$

With the help of the transformations (4) the corresponding partial slip boundary conditions (10)- (11) become

$$
\begin{gathered}
F(0)=\lambda\left[F^{\prime}(0)-L F(0) F^{\prime}(0)\right], \quad G(0)=\eta\left[G^{\prime}(0)-2 L F(0) G^{\prime}(0)\right], \quad H(0)=0 \\
F(\infty) \rightarrow 0, \quad G(\infty) \rightarrow 1 .
\end{gathered}
$$

The governing equations are still equations (5)- (7). The boundary conditions (9) are replaced by the partial slip boundary conditions (13). It is clear that the boundary conditions at infinity remain unaltered.

\section{Numerical solution of the problem}

We solve the system of nonlinear differential Eqns. (5)- (7) under the boundary conditions (13) by adopting the same second order numerical scheme described in our previous works $[4,5,26]$. The semi-infinite integration domain $\zeta \in[0, \infty)$ is replaced by a finite domain $\zeta \in\left[0, \zeta_{\infty}\right)$. In practice, $\zeta_{\infty}$ should be chosen sufficiently large so that the numerical solution closely approximates the terminal boundary conditions.

Now, suppose we introduce a mesh defined by

$$
\zeta_{i}=i h(i=0,1, \ldots n)
$$

$h=0.05$ being the mesh size, and discretize equations (5)- (7) using the central difference approximations for the derivatives, then the following equations are 
obtained.

$$
\begin{gathered}
\frac{F_{i+1}-2 F_{i}+F_{i-1}}{h^{2}}-H_{i}\left(\frac{F_{i+1}-F_{i-1}}{2 h}\right)-F_{i}^{2}+G_{i}^{2}-F_{i}-\frac{1}{2} L\left[\left(\frac{F_{i+1}-F_{i-1}}{2 h}\right)^{2}\right. \\
\left.-3\left(\frac{G_{i+1}-G_{i-1}}{2 h}\right)^{2}-2 F_{i}\left(\frac{F_{i+1}-2 F_{i}+F_{i-1}}{h^{2}}\right)\right]-1=0 \\
\frac{G_{i+1}-2 G_{i}+G_{i-1}}{h^{2}}-H_{i}\left(\frac{G_{i+1}-G_{i-1}}{2 h}\right)-2 F_{i} G_{i}-G_{i} \\
+L\left[\left(\frac{F_{i+1}-F_{i-1}}{2 h}\right)\left(\frac{G_{i+1}-G_{i-1}}{2 h}\right)+F_{i}\left(\frac{G_{i+1}-2 G_{i}+G_{i-1}}{h^{2}}\right)\right]=0, \\
H_{i+1}=H_{i}-h\left(F_{i}+F_{i+1}\right) .
\end{gathered}
$$

Note that Equations (15) and (16), which are written at the $i^{\text {th }}$ mesh point, the first and second derivatives are approximated by the central differences centered at the $i^{\text {th }}$ mesh point, while in Equation (17), which is written at the $(i+1 / 2)^{t h}$ mesh point, the first derivative is approximated by the difference quotient at the $i^{t h}$ and $(i+1)^{t h}$ mesh points and the right hand sides are approximated by the respective averages at the same two mesh points. This scheme ensures to preserve the second order accuracy of the spatial discretization.

Equations (15) and (16) are three term recurrence relations in $F$ and $G$ respectively. Hence, in order to start the recursion, besides the values of $F_{0}$ and $G_{0}$ the values of $F_{1}$ and $G_{1}$ are also required. These values can be obtained by Taylor series expansion near $\zeta=0$ for $F$ and $G$.

If

$$
F^{\prime}(0)=s_{1}, \quad G^{\prime}(0)=s_{2},
$$

we have

$$
\begin{aligned}
& F_{1}=F(0)+h F^{\prime}(0)+\frac{h^{2}}{2} F^{\prime \prime}(0)+O\left(h^{2}\right) \\
& G_{1}=G(0)+h G^{\prime}(0)+\frac{h^{2}}{2} G^{\prime \prime}(0)+O\left(h^{2}\right)
\end{aligned}
$$

The values $H(0)$ and $G(0)$ are given as boundary conditions in Equation (13). The values $F^{\prime \prime}(0)$ and $G^{\prime \prime}(0)$ can be obtained directly from Equations (6) and (7). After obtaining the values of $F_{1}$ and $G_{1}$ the integration can now be performed as follows. $H_{1}$ can be obtained from Equation (17). Using the values of $H_{1}$ in Equations (15) and (16), the values of $F_{2}$ and $G_{2}$ are obtained. At the next cycle, $H_{2}$ is computed from Equation (17) and is used in Equations (15) and (16)to obtain $F_{3}$ and $G_{3}$ respectively. The order indicated above is followed for the subsequent cycles. The integration is carried out until the values of $F$, 
$G$ and $H$ are obtained at all the mesh points. Note that we need to satisfy the two asymptotic boundary conditions (Eq. (13)). In fact, $s_{1}$ and $s_{2}$ are found by shooting method along with fourth order Runge-Kutta method so as to fulfil the free boundary conditions at $\zeta=\zeta_{\infty}$ in Equation (9).

A noteworthy fact is that for the same values of the flow parameters, the value of the numerical infinity should be chosen larger $\left(\zeta_{\infty}=27\right)$ as compared to the previously studied Karman flow problem $\left(\zeta_{\infty}=10\right)$ [5], so that the numerical solution closely approximates the terminal boundary conditions at $\zeta_{\infty}$.

\section{Results and discussions}

The value of $\zeta_{\infty}=27$, the numerical infinity has been taken larger as compared to the previous problem [5] $\left(\zeta_{\infty}=10\right)$ and kept invariant through out the run of the program. Although, the results are shown only from the disk surface $\zeta=0$ to $\zeta=14$, the numerical integrations were performed over a substantially larger domain in order to assure that the outer asymptotic boundary conditions are satisfied. To validate our approach, the values of $F, G$ and $H$ for no-slip condition $(\lambda=0)$ are compared with those reported by Owen and Rogers [21] for a viscous fluid $(L=0)$ and reported in Table-1. The present numerical results presented herein confirm the earlier calculations of Owen and Rogers [21] for the three velocitv comnonents.

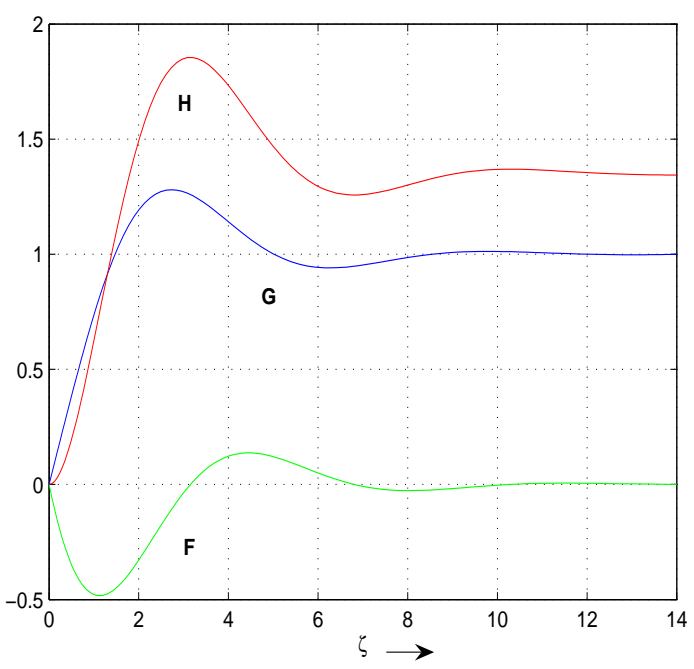

Fig. 2. Velocity profile for the Newtonian flow for $\lambda(=\eta)=0$.

Figure 2 depicts that near the disk, the radial component of the velocity $F$ is radially inwards. It may be of interest to note that this radially inward flow is the cause of the accumulation of the tea leaves at the center of a stirred cup of tea. The behaviors of the three velocity components confirm the sketch shown in Figure 1. From the profile of $G$, we can deduce the value 
of the boundary layer thickness $\zeta_{99}$, which is defined as the axial coordinate for which the tangential velocity of the fluid is equal to the tangential fluid velocity at infinity within $1 \%$. For $L=0$ and $\lambda=0$, the classical value $\zeta_{99}=8$ is recovered by the present approach [21].

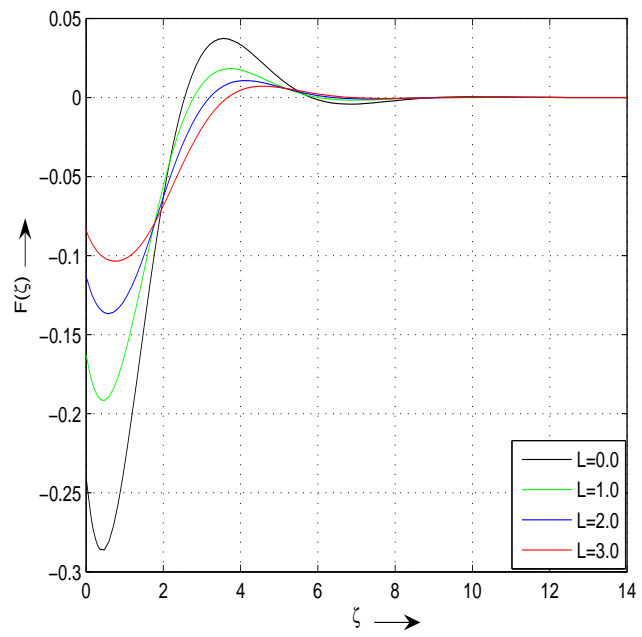

Fig. 3. Variation of $F$ with $L$ at $\lambda(=\eta)=1.0$.

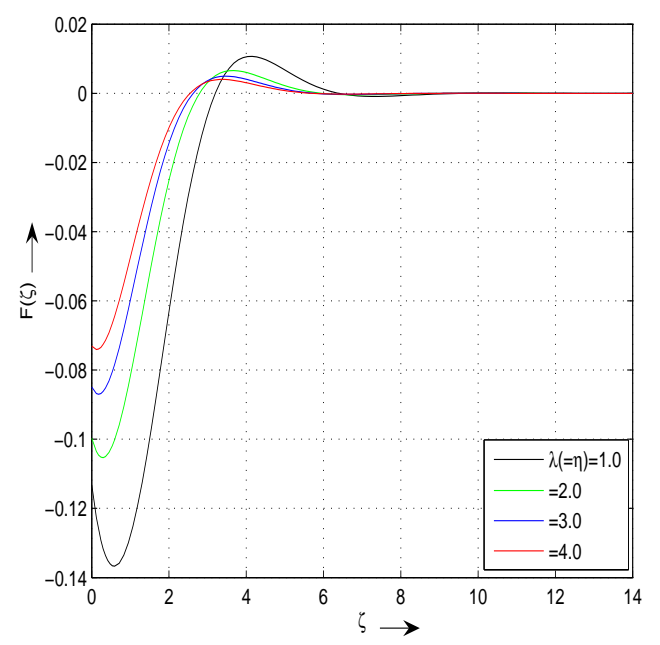

Fig. 4. Variation of $F$ with $\lambda(=\eta)$ at $L=2.0$.

In order to have an insight of the flow characteristics, results are plotted graphically for the uniform roughness $(\lambda=\eta)$, and different values of the flow parameters. The variations of the radial component of the velocity, $F$ with the non-Newtonian cross-viscous parameter $L$ and the slip parameter $\lambda(=\eta)$ are shown in Figures 3 and 4 respectively, when the other flow parameters are kept constant. Figure 3 reveals that $L$ has a prominent effect on $F$, near the disk. The magnitude of the radial inflow, near the disk, decreases with an increase in $L$. The radial component of velocity, near the disk remains negative for all values of the non-Newtonian parameter $L$, reverses the direction away from the disk, and finally approaches its asymptotic value. Thus, cross-overs are found in the velocity profile. Figure 4 shows the variation of $F$ with the slip parameter $\lambda(=\eta)$, when other flow parameters are kept constant. It is clear that the effect of slip on $F$ is also prominent near the disk. The velocity profiles reverse the direction away from the disk and approach the asymptotic value at a shorter distance from the disk as compared to the former case.

Figures 5 and 6 depict the variations of the azimuthal component of the velocity with $L$ and $\lambda(=\eta)$ respectively. The non-Newtonian parameter $L$ has a spectacular effect on $G$, away from the disk (near $\zeta=2.0$ ), as is clear from Figure 5 . An increase in $L$ decreases the velocity profile $G$ near the disk. The velocity component increases (see Figure 6) near the disk with an increase in $\lambda(=\eta)$. Thus, it is interesting to find that the slip has an opposite effect to that of the cross-viscous parameter on $G$. From these profiles, the boundary layer thickness $\zeta_{99}$ can be calculated for all flow parameters. The variations of 


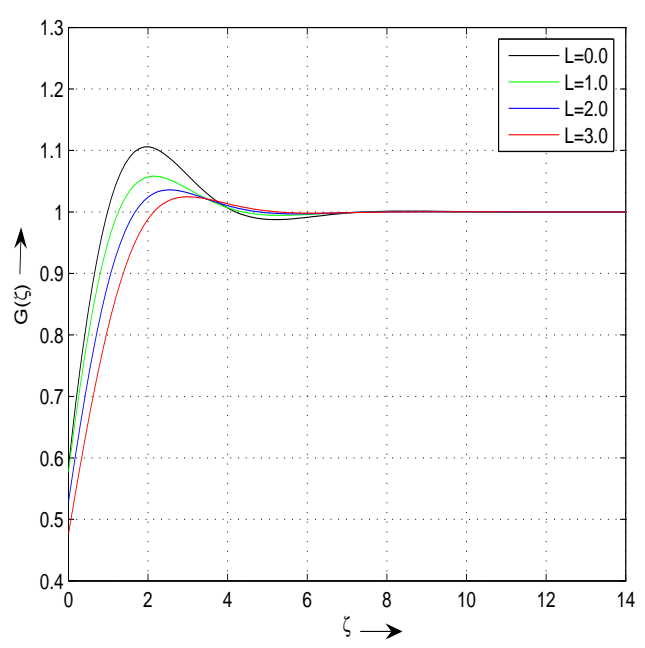

Fig. 5. Variation of $G$ with $L$ at $\lambda(=\eta)=1.0$.

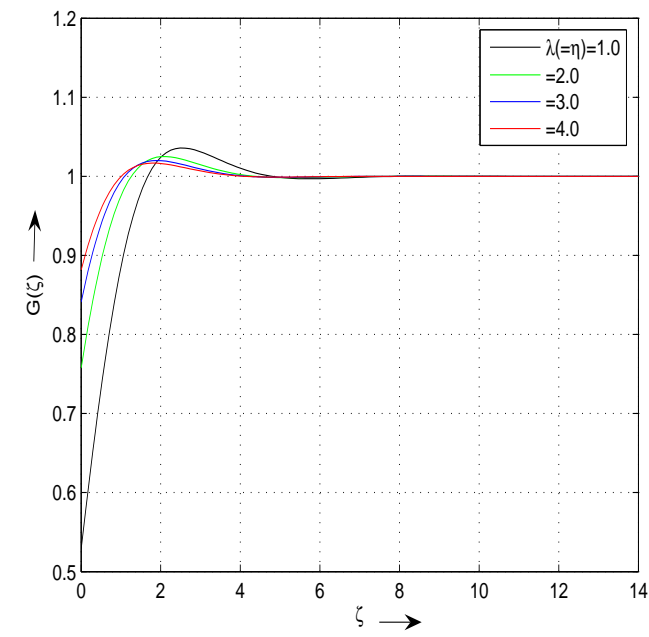

Fig. 6. Variation of $G$ with $\lambda(=\eta)$ at $L=2.0$.

$\zeta_{99}$ with the slip parameter $\lambda$ and the non-Newtonian parameter $L$ are shown in Figures 7 and 8 respectively and compared with the classical value $\zeta_{99}=8$ obtained for a Newtonian fluid $L=0$ and no slip condition $\lambda=0$. For $L=2$, $\zeta_{99}$ slightly increases with increasing values of the slip parameter $\lambda$, following the quadratical fitting law:

$$
\zeta_{99}=0.098 \lambda^{2}-0.061 \lambda+2.8
$$

For a given value of the slip parameter $\lambda=1, \zeta_{99}$ slightly increases also with increasing values of the non-Newtonian parameter $L$, following also a quadratical law:

$$
\zeta_{99}=0.055 L^{2}-0.027 L+3.9
$$

It is noteworthy that the values of $\zeta_{99}$ are much weaker than the one obtained for no slip condition $(\lambda=0)$ in the case of a Newtonian fluid $(L=0): \zeta_{99}=8$ $[21]$.

Another interesting quantity, which can be deduced from the profiles of $\mathrm{F}$ and $\mathrm{G}$, is the turning moment for the disk with fluid on both sides. The expression of the dimensionless moment coefficient $C_{M}$ is given by:

$$
C_{M}=\frac{-2 \pi G^{\prime}(0)[1-2 L F(0)]}{\sqrt{R e}}
$$

with $R e=\Omega R^{2} / \nu$ the rotational Reynolds number based on the disk radius $R$ 


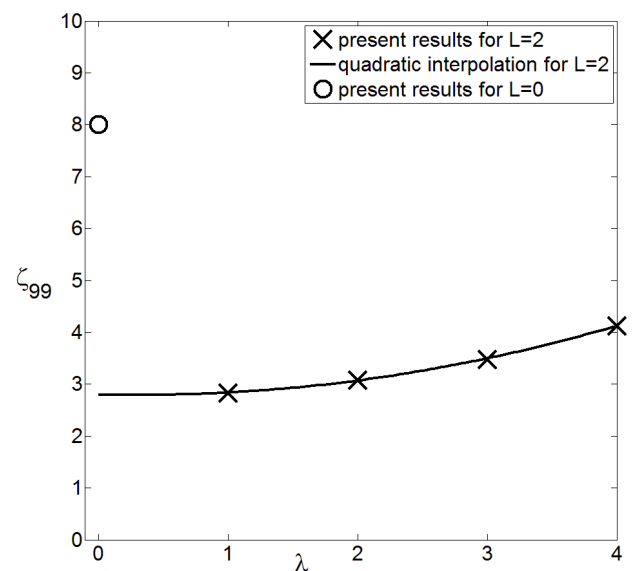

Fig. 7. Variation of the boundary layer thickness $\zeta_{99}$ with $\lambda$ for $L=2$.

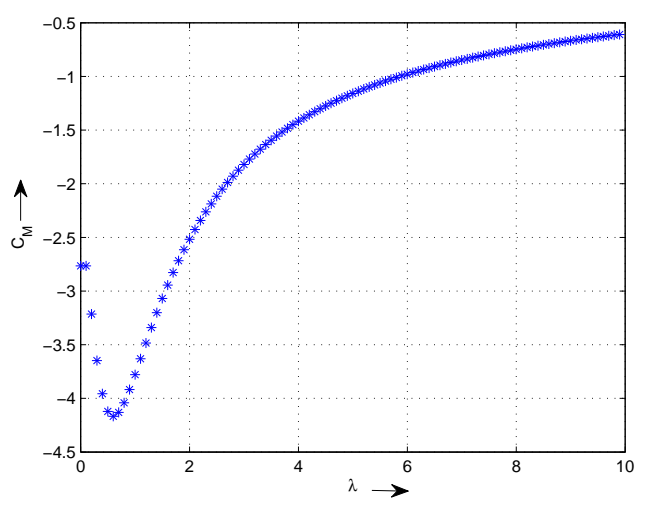

Fig. 9. Variation of the moment coefficient $C_{M}$ with $\lambda$ for $L=1$ and $R e=1$.

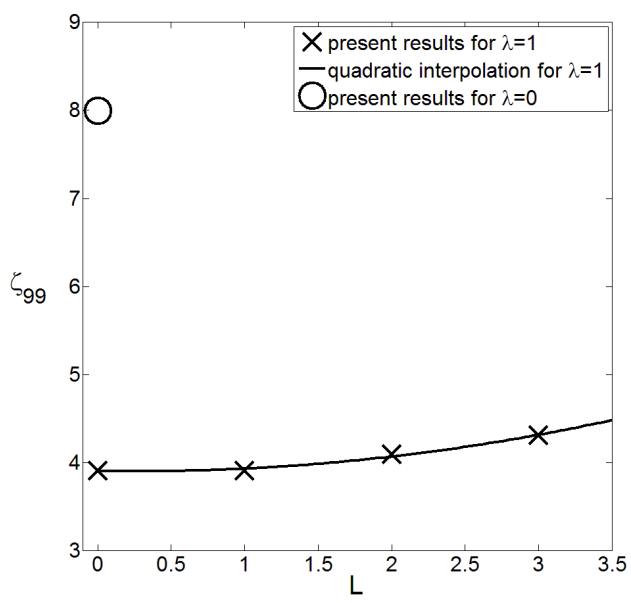

Fig. 8. Variation of the boundary layer thickness $\zeta_{99}$ with $L$ for $\lambda=1$.

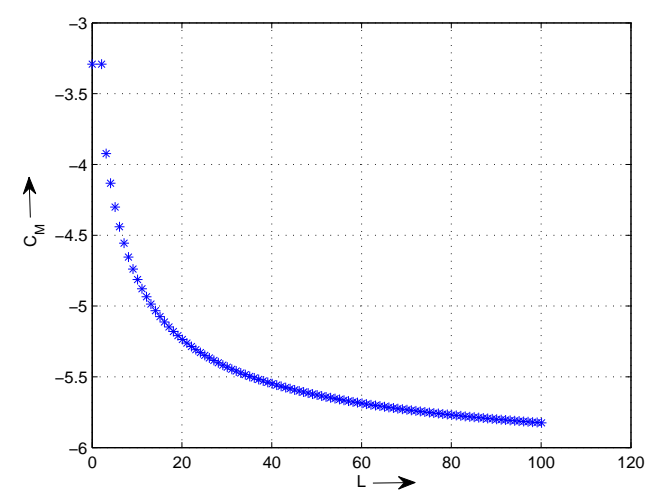

Fig. 10. Variation of the moment coefficient $C_{M}$ with $L$ for $\lambda=1$ and $R e=1$.

and the maximum velocity $(\Omega R)$. This definition of $C_{M}$ is the extension to the finite disk problem, which supposes that the disk radius is large enough. For $L=0$, Equation (21) reduces to $C_{M}=-2 \pi G^{\prime}(0)$. In that case, the moment coefficient depends only on $G^{\prime}(0)$. Note that the present value of $G^{\prime}(0)$ for a Newtonian fluid and no slip condition $(L=\lambda=0)$ is in excellent agreement with the value $G^{\prime}(0)=0.77289$ obtained by Owen and Rogers [21]. Thus, we got the classical value $C_{M}=-4.86$ in that basic case.

The variations of $C_{M}$ with the slip $\lambda$ and non-Newtonian $L$ parameters are shown in Figures 9 and 10 respectively. Whatever the flow parameters, $C_{M}$ exhibits negative values. It may be attributed to the flow problem: a rotating fluid at infinity over a stationary disk. Thus, the axial gradient of the tangential velocity $G^{\prime}$ is still positive and $C_{M}$ is then always negative. The Von Kármán flow considered by Sahoo [5] is precisely the inverse problem, which explains the different signs. For $L=1$ and $R e=1$ (see Fig.9), the moment 
coefficient $C_{M}$ in absolute values strongly increases (resp. decreases) with increasing values of the slip parameter $\lambda$ for $\lambda<0.6$ (resp. $\lambda>0.6$ ). It tends rapidly to zero for high values of $\lambda$, which means that the torque required to maintain the disk at its original speed is almost zero when the slip parameter is high. Omitting the different boundary conditions between the two problems, the present results confirm the previous ones of Sahoo [5] for Von Kármán flows and comparable values are obtained.

The slip parameter $\lambda$ is now fixed to unity and the non-Newtonian parameter $L$ varies between 0 and 100 for $R e=1$ (Fig.10). $C_{M}$ increases in magnitude with the parameter $L$. In that case, the torque required to maintain the disk at rest is much higher than those necessary to maintain the disk at $\Omega$ for the Von Kármán flow considered by Sahoo [5].

The variations of the axial component of the velocity $H$ with $L$ and $\lambda(=\eta)$ have been plotted in Figures 11 and 12 respectively. The figures show that both parameters have a similar effect on $H$. It is clear that the axial velocity becomes flatter with an increase in $L$ and $\lambda(=\eta)$. The axial velocity at infinity $H_{\infty}$ in both cases is strongly reduced compared to the basic case $L=0$ and $\lambda=0$, for which $H_{\infty} \simeq 1.3494$.

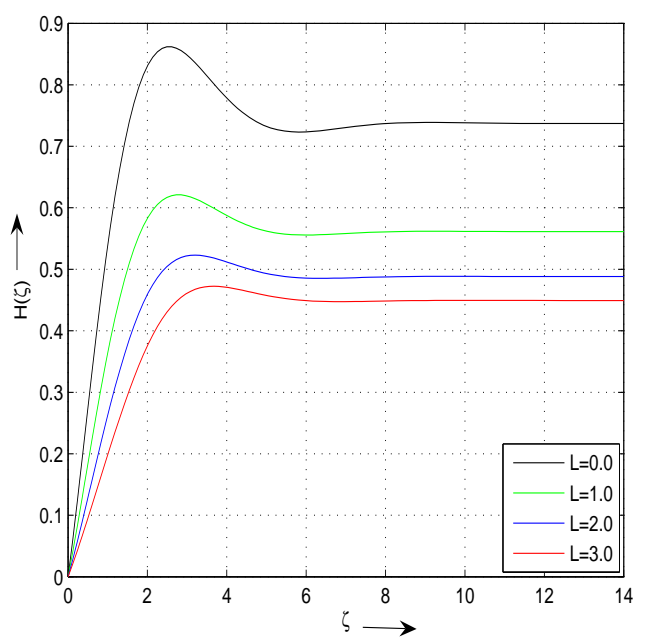

Fig. 11. Variation of $H$ with $L$ at $\lambda(=\eta)=1.0$.

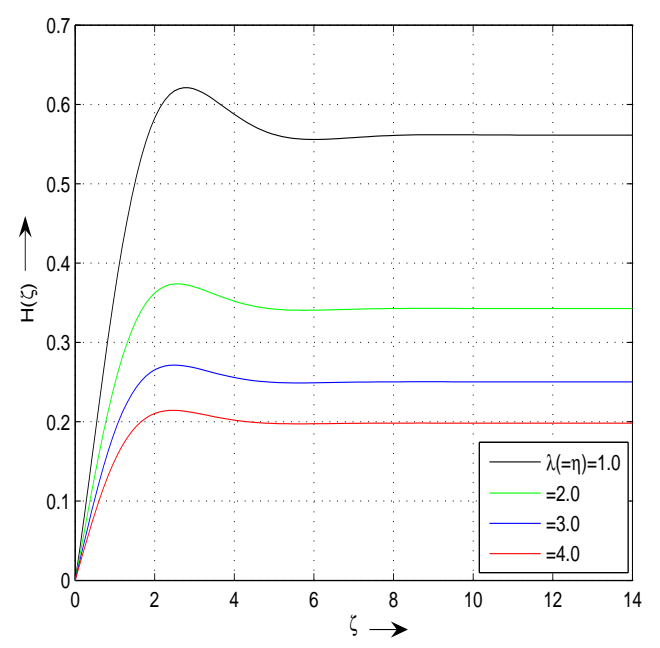

Fig. 12. Variation of $H$ with $\lambda(=\eta)$ at $L=2.0$. 
Table 1

Variations of $F, G, H$ for $L=0$ and $\lambda(=\eta)=0$.

\begin{tabular}{ccccccc}
\hline \hline & $F$ & \multicolumn{3}{c}{$G$} & \multicolumn{2}{c}{$H$} \\
\hline$\zeta$ & Current result & Owen \& Rogers [21] & Current result & Owen \& Rogers [21] & Current result & Owen \& Rogers [21] \\
\hline 0.0 & 0.000000 & 0.0000 & 0.000000 & 0.0000 & 0.000000 & 0.0000 \\
0.5 & -0.348650 & -0.3487 & 0.383430 & 0.3834 & 0.194373 & 0.1944 \\
1.0 & -0.478766 & -0.4788 & 0.735429 & 0.7354 & 0.624103 & 0.6241 \\
1.5 & -0.449633 & -0.4496 & 1.013401 & 1.0134 & 1.098743 & 1.0987 \\
2.0 & -0.328745 & -0.3287 & 1.192367 & 1.1924 & 1.492875 & 1.4929 \\
2.5 & -0.176206 & -0.1762 & 1.272136 & 1.2721 & 1.745869 & 1.7459 \\
3.0 & -0.036086 & -0.0361 & 1.271405 & 1.2714 & 1.849641 & 1.8496 \\
3.5 & 0.066310 & 0.0663 & 1.218219 & 1.2182 & 1.830807 & 1.8308 \\
9.5 & -0.010216 & -0.0102 & 1.011849 & 1.0118 & 1.361698 & 1.3617 \\
10.0 & -0.003282 & -0.0033 & 1.012120 & 1.0121 & 1.368328 & 1.3683 \\
10.5 & 0.001819 & 0.0018 & 1.009906 & 1.0099 & 1.368882 & 1.3689 \\
11.0 & 0.004738 & 0.0047 & 1.006537 & 1.0065 & 1.365423 & 1.3654 \\
11.5 & 0.005681 & 0.0057 & 1.003090 & 1.0031 & 1.360067 & 1.3601 \\
12.0 & 0.005170 & 0.0052 & 1.000271 & 1.0003 & 1.354546 & 1.3545 \\
12.5 & 0.003827 & 0.0038 & 0.998411 & 0.9984 & 1.350003 & 1.3500 \\
20.0 & 0.000102 & - & 0.999893 & - & 1.349325 & - \\
25.0 & 0.000009 & - & 1.000014 & - & 1.349457 & - \\
25.5 & 0.000011 & - & 1.000007 & - & 1.349447 & - \\
26.0 & 0.000010 & - & 1.000001 & - & 1.349437 & - \\
26.5 & 0.000008 & - & 0.999997 & - & 1.349428 & - \\
$\infty$ & 0.000000 & 0.0000 & 1.000000 & 1.0000 & 1.349424 & 1.3494 \\
\hline
\end{tabular}




\section{Conclusions}

In this work, the slip flow due to the rotation of a non-Newtonian Reiner-Rivlin fluid near a stationary disk have been examined precisely for the first time. The resulting highly nonlinear differential equations are solved by adopting a second order numerical scheme. The combined effects of the slip $(\lambda=\eta)$ and the non-Newtonian parameter $(L)$ on the velocity field are studied in detail. It is interesting to find that $\lambda(=\eta)$ and $L$ have similar effects on the radial and axial velocity components. For increasing values of $\lambda$ or $L$, a slight thicknening of the boundary layer on the stationary disk is observed. On the other hand, both the parameters have opposite effects on the azimuthal component of the velocity and the moment coefficient. Thus, the torque required to maintain the disk at rest tends to zero for increasing values of $\lambda$ and increases (in absolute values) with $L$ showing a predominant influence of the non-Newtonian parameter.

\section{Acknowledgements}

The authors express their very sincere thanks to Prof. H.I. Andersson, Department of Energy and Process Engineering, NTNU, Norway for his valuable suggestions.

\section{References}

[1] A.C. Srivastava and G.C. Sarma. Forced flow of a second-order fluid against a rotating disk. Int. J. Non-Linear Mech., 5:525-532, 1970.

[2] H.I. Andersson, E. de Korte, and R. Meland. Flow of a power-law fluid over a rotating disk revisited. Fluid Dyn. Res., 28:75-88, 2001.

[3] H.A. Attia. Numerical study of flow and heat transfer of a non-Newtonian fluid on a rotating porous disk. Appl. Math. Comp., 163:327-342, 2005.

[4] B. Sahoo and H.G. Sharma. Effects of partial slip on the steady Von Kármán flow and heat transfer of a non-Newtonian fluid. Bull. Braz. Math. Soc., 38:595$609,2007$.

[5] B. Sahoo. Effects of partial slip, viscous dissipation, Joule heating on Von Kármán flow and heat transfer of an electrically conducting non-Newtonian fluid. Comm. Nonlin. Sci. Num. Sim., doi:10.1016/j.cnsns.2008.10.021, 2010.

[6] U.T. Bödewadt. Die Drehströmung über festem Grund. J. Angew. Math. Mech., ZAMM, 20:241-253, 1940. 
[7] J.A.D. Ackroyd. On the steady flow produced by a rotating disc with either surface suction or injection. J. Engng. Maths., 12(3):207-220, 1978.

[8] W.G. Cochran. The flow due to a rotating disk. Proc. Camb. Phil. Soc., 30:365375, 1934.

[9] H. Schlichting. Boundary layer theory (7th Edition). McGraw-Hill, 1979.

[10] G.K. Batchelor. Note on a class of solutions of the Navier-Stokes equations representing steady non rotationally symmetric flow. Quart. J. Mech. Appl. Math., 4:29-41, 1951.

[11] D.M. Hannah. Forced flow against a rotating disc. ARC-RM-2772, 1952.

[12] K. Stewartson. On the flow between two rotating coaxial disks. Proc. Camb. Phil. Soc. Math. Phys. Sci., 49:333-341, 1953.

[13] H.E. Fettis. On the integration of a class of differential equations occuring in boundary layer and other hydrodynamic problems. In Proc. Fourth Midwestern Conf. on Fluid Mech., pages 93-114, Purdue, 1955.

[14] M.H. Rogers and G.N. Lance. The rotationally symmetric flow of a viscous fluid in the presence of an infinite rotating disk. J. Fluid Mech., 7:617-631, 1960.

[15] E.W. Schwiderski and H.J. Lugt. Rotating flows of Von kármán and Bödewadt type. Phys. Fluids, 7:867-872, 1964.

[16] E.W. Schwiderski and H.J. Lugt. Rotating flows over a rotating disk. Ing. Archiv, 34:198-206, 1965.

[17] R.S. Nanda. Revolving flow of an incompressible fluid past a porous flat plate. J. Sci. Engng. Res., 5:59-64, 1960.

[18] J.E. Nydahl. Heat transfer for the Bödewadt problem. PhD thesis, Colorado State University, 1971.

[19] M.H. Rogers and G.N. Lance. The boundary layer on a disk of finite radius in a rotating fluid. Quart. J. Mech. Appl. Math., 17:319-330, 1964.

[20] S.S. Chawala and R. Purushothaman. Spin-up in the Bödewadt flow. J. Math. Anal. Appl., 71:463-481, 1979.

[21] J.M. Owen and R.H. Rogers. Flow and heat transfer in rotating disk systems. Vol. 1: Rotor-Stator Systems. Research Stud. Presss Ltd, John Wiley \& Sons, 1989.

[22] S.S. Chawla and P.K. Srivastava. On the Bödewadt-Rosenblat flow. Z. Angew. Math. Phys., ZAMP, 57:793-814, 2006.

[23] B. Sharon and O. MacKerrel. Stability of Bödewadt flow. Phil. Trans. Roy. Soc., 363:1181-1187, 2010.

[24] C.W. Kitchens and T.S. Chang. Newtonian and non-newtonian liquids rotating adjacent to a stationary surface. Appl. Sci. Res., 27:283-296, 1973. 
[25] Th. Von Kármán. Über laminare und turbulente Reibung. Z. Angew. Math. Mech., ZAMM, 1:233-252, 1921.

[26] B. Sahoo. Flow and heat transfer of an electrically conducting third grade fluid past an infinite plate with partial slip. Meccanica, Accepted, 2009. 